\title{
La Red Universitaria de la Ruta de Integración Latinoamericana (UniRila) y el Corredor Bioceánico: relaciones culturales entre Porto Murtinho/Brasil y Carmelo Peralta/Paraguay
}

\author{
A Rede de Universidades da Rota de Integração Latino-Americana (UniRila) \\ e o Corredor Bioceânico: relações culturais entre Porto Murtinho/Brasil e \\ Carmelo Peralta/Paraguai
}

\begin{abstract}
The University Network on the Latin American Integration Route (UniRila) and the Bioceanic Corridor: cultural relations between Porto Murtinho/Brasil and Carmelo Peralta/Paraguay
\end{abstract}

\author{
Hanae Caroline Quintana Shiota ${ }^{1}$ \\ Mariana de Barros Casagranda Akamine ${ }^{1}$ \\ Alessandra Chaia $^{1}$ \\ Érika Santos Silva ${ }^{1}$
}

Recibido el 1 de jul. de 2021; revisado e aprobado el 17 de sept. de 2021; aceptado el 13 de oct. de 2021 DOI: http://dx.doi.org/10.20435/inter.v22i4.3429

\begin{abstract}
Resumen: La consolidación y la articulación entre universidades abre la posibilidad de un espacio privilegiado para discusiones, reflexiones y construcción de propuestas significativas de acciones académicas que posibiliten el trabajo técnico-científico interdisciplinario. La Red de Universidades en la Ruta de la Integración Latinoamericana (UniRila) fue creada en 2016, con el propósito de desarrollar acciones que apoyen los estudios de viabilidad del Corredor Bioceánico entre Brasil, Paraguay, Argentina y Chile. Este trabajo procura analizar la identidad y relación cultural entre Porto Murtinho-Brasil y Carmelo Peralta-Paraguay con vistas a las acciones de UniRila, enfatizando que la cultura se guió por principios y valores que sostienen el paradigma cultural de un pueblo en sus hábitos, creencias, valores e identidades, bien como la forma de difusión y conservación. La investigación utilizó el método deductivo con alcance sistémico mediante búsqueda bibliográfica y documental. Por tanto, para comprender mejor los temas relacionados con la cultura y los procesos de identidad, es necesario traer una breve noción de qué es la cultura y cuál es la relación cultural entre Porto Murtinho y Carmelo Peralta con vistas a la implementación del Corredor Bioceánico. Se infiere que la relación establecida conduce a la observación de que el entrelazamiento cultural estará en constante mutación o transformación a medida que se produzca la interacción e integración social.
\end{abstract}

Palabras clave: Ruta Bioceánica; UniRila; cultura; Brasil; Paraguay.

Resumo: A consolidação e a articulação entre universidades abrem a possibilidade de um espaço privilegiado para discussões, reflexões e construções de propostas significativas de ações acadêmicas para viabilização de trabalhos técnico-científicos interdisciplinares. A Rede de Universidades da Rota de Integração LatinoAmericana (UniRila) foi criada em 2016, com o propósito de desenvolver ações que dessem suporte aos estudos de viabilização do Corredor Bioceânico entre o Brasil, Paraguai, Argentina e Chile. O presente trabalho busca analisar a relação identitária e cultural existente entre Porto Murtinho-Brasil e Carmelo PeraltaParaguai com vistas às ações da UniRila, enfatizando que a cultura é norteada por princípios e valores que sustentam o paradigma cultural de um povo em seus hábitos, crenças, valores e identidades e pela forma de difusão e preservação. A pesquisa utilizou o método dedutivo com uma abrangência sistêmica por meio de levantamento bibliográfico e documental. Assim, para que se possa compreender melhor as questões referentes à cultura e aos processos identitários, antes, é preciso trazer uma breve noção do que é cultura, qual a relação cultural existente entre Porto Murtinho e Carmelo Peralta com vistas à implantação do Corredor Bioceânico. Infere-se que a relação estabelecida leva à constatação de que o entrelaçamento cultural estará em constante mutação ou transformação à medida que a interação e a integração social ocorrerem.

Palavras-chave: Rota Bioceânica; UniRila; cultura; Brasil; Paraguai.

Abstract: Consolidation and articulation between universities open up a privileged space for possible discussions, reflections and construction of significant proposals for academic actions to enable

\footnotetext{
${ }^{1}$ Universidad Católica Dom Bosco (UCDB), Campo Grande, Mato Grosso do Sul, Brasil.
} 
interdisciplinary technical-scientific work. The University Network on the Latin American Integration Route (UniRila) was created in 2016, with the purpose of developing actions that support feasibility studies for the Bioceanic Corridor between Brazil, Paraguay, Argentina and Chile. This paper intends to analyze the identity and cultural relationship between Porto Murtinho-Brasil and Carmelo Peralta-Paraguay with actions of UniRila perspective, emphasizing the principles and values that culture is guided by, to support a people's cultural paradigm in their habits, beliefs, values and identities besides cultural diffusion and preservation. The research used the deductive method with a systemic scope through bibliographic and documentary surveys. Therefore, in order to better understand issues related to culture and identity processes, it is necessary to briefly note what is culture and the cultural relationships between Porto Murtinho and Carmelo Peralta as the Bioceanic Corridor is consolidated. It is inferred that the established relationship leads to the observation that the cultural intertwining will be in constant mutation or transformation as the interaction and social integration occur.

Keywords: Bioceanic Route; UniRila; culture; Brazil; Paraguay.

\section{INTRODUCCIÓN}

El objetivo principal del actual trabajo es presentar las relaciones culturales de los territorios fronterizos e investigar cómo rodea los países vecinos Brasil y Paraguay, puesto que ambos forman parte de las acciones de la Red Universitaria de Integración Latinoamericana (UniRila), con vistas a la implantación del Corredor Bioceánico. Este puede considerarse una palanca para el desarrollo local y la promoción de un gran flujo de personas y de insumos, afectando así la formación y la construcción cultural de las ciudades pertenecientes a los cuatro países por los que pasará: Argentina (AR), Chile (CL), y específicamente Brasil (BR) y Paraguay (PY), siendo las ciudades fronterizas Porto Murtinho (BR) y Carmelo Peralta (PY) los enfoques de esta investigación.

El Corredor Bioceánico puede verse como un espacio físico de paso, mientras que la

"ruta"se traduce como un eje de integración humana, social y cultural que va más allá de la idea económica, logística y de transporte que se producirá naturalmente a partir de su implementación.

En este contexto, con la implementación del Corredor Bioceánico prevista de 2022 a 2023, las investigaciones realizadas por la UniRila posibilitan la construcción de un espacio privilegiado de discusión, reflexión y preservación de diversos temas sociales, ambientales, económicos, entre los que destacan la cultura y sus diferentes conceptos, así como los medios utilizados para preservar la identidad local y la cultura del pueblo.

Para ello, el actual estudio se vale del método deductivo, con alcance sistémico por medio de la búsqueda bibliográfica y documental, con la intención de profundizar los conocimientos sobre el Corredor Bioceánico, de investigar las prácticas culturales y de identidad brasileñas y paraguayas y, entonces, analizar la fuerte relación que ya existe entre las ciudades fronterizas, así como su mayor acercamiento cultural tras la conclusión de la ruta.

\section{EL CORREDOR BIOCEÁNICO: CONTEXTO HISTÓRICO EN LA BÚSQUEDA DE LAS RELACIONES CULTURALES}

Hace un poco más de dos décadas, todavía en el gobierno de Fernando Henrique Cardoso (1995-2003), junto a los demás gobiernos de países que forman frontera con Brasil, fue lanzada la Iniciativa de la Integración de la Infraestructura Regional Sudamericana (IIRSA), con el objetivo de cooperación estatal e integración del continente sudamericano, para la creación de un corredor que posibilitara la destinación de la producción de dichos países. 
El 21 de diciembre de 2015, los gobiernos de Brasil, Argentina, Chile y Paraguay aprobaron la Declaración de Asunción, comprendiendo acciones que posibilitaron la implantación del Corredor Bioceánico conectando la ciudad de Porto Murtinho, en la provincia de Mato Grosso de Sul (MS) a los puertos del norte de Chile.

El Corredor tiene como objetivo conectar el Océano Atlántico al Pacífico, para la destinación de la producción del Puerto de Santos, en la provincia de Sao Paulo (SP), pasando por el territorio de Mato Grosso do Sul, MS, y cruzará el municipio de Campo Grande (MS), hasta la frontera brasileña en la ciudad de Porto Murtinho (MS). Alcanzando, de esta forma, tierras paraguayas en la ciudad de Carmelo Peralta. Ya en territorio internacional cruzará diversas ciudades paraguayas, argentinas y chilenas, hasta los puertos de Antofagasta, Mejillones e Iquique, en Chile.

Con respecto a la nomenclatura de términos utilizados, Campos y Faria (2020) destacan que la Ruta Bioceánica, o Ruta de Integración Latino Americana (RILA), es un corredor vial, el cual hará posible el acortamiento de la distancia para la reducción del tiempo de las exportaciones, bien como de las importaciones brasileñas entre los mercados de Asia, Oceanía y Norteamérica.

El sitio web del Gobierno del Estado de Mato Grosso do Sul (2019) destaca cinco objetivos del corredor bioceánico: la reducción del tiempo de tránsito y del coste del servicio de transporte, almacenaje e inventario; el estímulo del uso de más de un modal; la generación de un flujo de carga y pasajeros eficiente, en términos de confiabilidad; previsibilidad y seguridad; el estímulo en la formación de sociedades y el desarrollo de proyectos; la integración productiva y la agregación de valor en los países de origen y de destino, bien como en los países de tránsito.

El proyecto en marcha tiene diferentes enfoques, teniendo en cuenta las particularidades de cada región que cruzará. En Brasil, específicamente en el estado de Mato Grosso do Sul, lo más destacado es posibilitar a la agroindustria un nuevo camino de destinación de la producción, así como facilitar la importación de insumos a precios más competitivos, como señala el Gobierno del Estado (MATO GROSSO DO SUL, 2019).

Cabe mencionar que cada gobierno de los países que forman parte de la Ruta persigue objetivos específicos para sus regiones. El gobierno chileno tiene como objetivo promover el comercio con los países de la región, afirmando al país como una importante plataforma logística. Argentina, por su parte, pretende fortalecer inversiones en infraestructura del orden de los US\$ 15 mil millones, que según el sitio web del Gobierno del Estado de Mato Grosso do Sul (2019), se invertirán a la medida que la ruta cruce las provincias de Salta y Jujuy. Para los paraguayos, el Corredor Bioceánico favorece la infraestructura e integración de la región del «Chaco»con el resto del país.

Asato, Gonçalves y Wilke (2019) subrayan que para Brasil, especialmente en el estado de Mato Grosso do Sul:

El Corredor Bioceánico será una palanca para el desarrollo de la ciudad de Porto Murtinho y de sus alrededores, impulsados por la ubicación estratégica del municipio, que favorecerá el turismo y, en paralelo, por la logística, que, a partir de la construcción de un Puente binacional que conectará la ciudad de Mato Grosso do Sul con Carmelo Peralta, en Paraguay, que se espera esté terminado a mediados de 2022. (ASATO; GONÇALVES; WILKE, 2019, p. 142, traducción libre de los autores).

Para Asato, Gonçalves y Wilke (2019, p. 142, traducción libre de los autores) “[...] los objetivos del Corredor son facilitar el trámite logístico entre los países sudamericanos, y fortalecer el turismo, además de unir a los pueblos del cono sur del continente". 
Los autores aún destacan que las relaciones culturales que implican esta integración van más allá de las preguntas económicas y territoriales, ya que se vuelcan a las relaciones de los ciudadanos de pueblos brasileños y paraguayos y, en este contexto, se relacionan con las cuestiones de identidad, afectividad y al sentimiento de pertenencia.

Estas expresiones brindan un diálogo según Vieira, Vieira y Knopp (2010), con estudios que abordan cuestiones volcadas al territorio cultural y destacan que la sociedad ha sido testigo de diferentes escalas de globalización del mercado, no restringiéndose al espacio económico, sino que también incidiendo en la cultura, sociedad y relaciones políticas.

Para Lima (2013), los temas de memoria e identidad vinculados a la población de diferentes gobiernos municipales, y en particular a la ciudad de Porto Murtinho, MS, están relacionados con el fortalecimiento de la autoestima local y el orgullo de su historia, especialmente en lo que corresponde a edificios, testigos de las influencias de la colonización, de las multiculturalidades que sufrieron los territorios fronterizos, bien como los notables ciclos económicos que se dieron en el municipio.

A partir de la visión de Vieira, Vieira y Knopp (2010), y los estudios de Lima (2013) sobre la memoria y la identidad en la frontera Brasil-Paraguay, a través del municipio brasileño de Porto Murtinho, es posible afirmar la caracterización de una multiterritorialidad, es decir, múltiples territorios que conviven en un espacio plural en cuanto a cultura, identidad y economía, que se reafirma a través de la construcción del puente Brasil-Paraguay, trayendo profundos reflejos a las comunidades fronterizas.

De tal manera, la comprensión de los conceptos y las relaciones culturales entre BrasilParaguay son necesarias, en prominencia de la Ruta de Integración Latinoamericana (RILA).

\section{FRONTERAS SIMBÓLICAS: PUERTO CARMELO PERALTA-PY Y PORTO MURTINHO-BR}

Dentro de un contexto social y cultural, la noción de frontera se caracteriza como la idea de una Nación, y por lo tanto se desarrollan algunas discusiones sobre sus formas simbólicas. Cuando se piensa en el término "frontera", rápidamente se imagina una limitación que divide territorio, personas y entornos.

La frontera, entonces, denuncia las tensiones entre dos espacios: el interior y el exterior; el Yo y el Otro. Al mismo tiempo, promueve superposiciones entre sus pares binarios, lo que resulta en una zona híbrida donde se produce el contacto entre los polos separados, espacios en los que son puestos cara a cara. (SOUZA, 2014, p. 175, traducción libre de los autores).

Souza (2014) compara las fronteras como puentes en los que las poblaciones vecinas desarrollan una "[...] articulación ambulante y ambivalente» en la que ambos comparten un intersticio. La frontera en el plano geográfico es capaz de delimitar el espacio cultural de cada grupo, en cambio no es capaz de impedir que exista, de hecho, una articulación entre las culturas vecinas.

Pesavento (2002) informa sobre las dimensiones simbólicas que limitan la representación de la realidad cultural de cada pueblo, y distingue cómo cada individuo vive su sociedad local, cómo se porta en su grupo y desarrolla su culturalidad.

Todos sabemos que las fronteras, antes de ser hitos físicos o naturales, son sobre todo simbólicas. Son hitos, sí, pero sobre todo, referentes mentales que orientan la percepción de la realidad. En este sentido, son productos de esta capacidad mágica de representar 
el mundo a través de un paralelo de signos a través del cual los hombres se perciben y se califican a sí mismos, al cuerpo social, al espacio y al propio tiempo. Nos referimos al imaginario, este sistema de representaciones colectivas que da sentido a lo real y que guía los valores y la conducta. De esta manera, las fronteras son, sobre todo, culturales, es decir, son construcciones de sentido, siendo parte del juego social de representaciones que establecen clasificaciones, jerarquías, límites, orientando la mirada y apreciación sobre el mundo. (PESAVENTO, 2002, p. 35, traducción libre de los autores).

La frontera tiene como principal factor de representación de la realidad, y los significados culturales que se establecieron en orden jerárquico, por lo que sus clasificaciones sociales se han orientado hacia la construcción de la identidad. Se le da el nombre de "frontera simbólica» a quienes actúan como mediadores socioculturales entre el Yo y el Otro. Las fronteras son necesarias para distinguir las diferencias culturales, pero sin esa delimitación, la idea sería de un modelo cultural único.

Al pensar en fronteras simbólicas, encontramos una íntima relación con el pensamiento de Anderson Benedict (2008) en su obra Comunidades imaginadas, donde reporta la frontera como productora del sentimiento de pertenencia y a través de la cual uno de los pilares de la identidad se desarrolla. Pues bien, es en la frontera donde el territorio se divide y se unifica imperceptiblemente.

En la concepción moderna, la soberanía del Estado opera de manera integral, definitiva y homogénea sobre cada centímetro cuadrado de un territorio legalmente demarcado. Pero, en la imaginación más antigua, donde las provincias estaban definidas por centros, las fronteras eran porosas e indistintas, y las soberanías imperceptiblemente se desvanecían entre sí. (BENEDICT, 2008, p. 48, traducción libre de los autores).

Pesavento (2002) sostiene que la frontera sirve como puerta de entrada a una cultura diversificada. El sentido de pertenencia desarrollado dentro de la delimitación territorial es lo que genera las conexiones entre pueblos vecinos: entienden que provienen de culturas diferentes, pero al convivir de manera unificada, terminan desarrollando sus propias costumbres, caracterizando un pilar más de su identidad. Así siendo, el umbral de las fronteras simbólicas permite la aceptación y el rechazo simultáneos, que ocurren a todo momento. Como una vía de doble sentido que permite distintas posibilidades de tránsitos, la frontera permite movimientos simultáneos que incluso pueden resultar contradictorios: a veces los sujetos son bienvenidos, a veces son rechazados por los mismos motivos.

[...] incluso en esta dimensión de enfoque fijada por la territorialidad y la geopolítica, el concepto de frontera ya avanza hacia los dominios de esa construcción simbólica de pertenencia al que llamamos identidad y que corresponde a un marco de referencia imaginaria que se define por la diferencia. En esta medida, el concepto de frontera trabaja necesariamente con principios de reconocimiento que involucran analogías, oposiciones y correspondencias de igualdad, en un juego permanente de interpenetraciones y conexiones variadas. (PESAVENTO, 2002, p. 36, traducción libre de los autores).

En la ruta que albergará el Corredor Bioceánico en la provincia de Mato Grosso do Sul, Brasil, un claro ejemplo de frontera simbólica está compuesto por las ciudades de Porto Murtinho-BR y Carmelo Peralta-PY. Estas están divididas por el río Paraguay e interconectadas por carreteras, y es a través de estos medios de intersección que los residentes se desplazan por razones sociales, económicas y culturales. Las ciudades vecinas y fronterizas dividen los países Brasil y Paraguay: 
ambas mantienen la herencia cultural de sus países, pero desarrollan su identidad cultural de tal manera que están geográficamente delimitadas, pero unificadas por el sentimiento de pertenencia atribuido a las interacciones e integraciones del convivio diario.

Figura 1 - Frontera Brasil-Paraguay Porto Murtinho-Carmelo Peralta (sin escala)

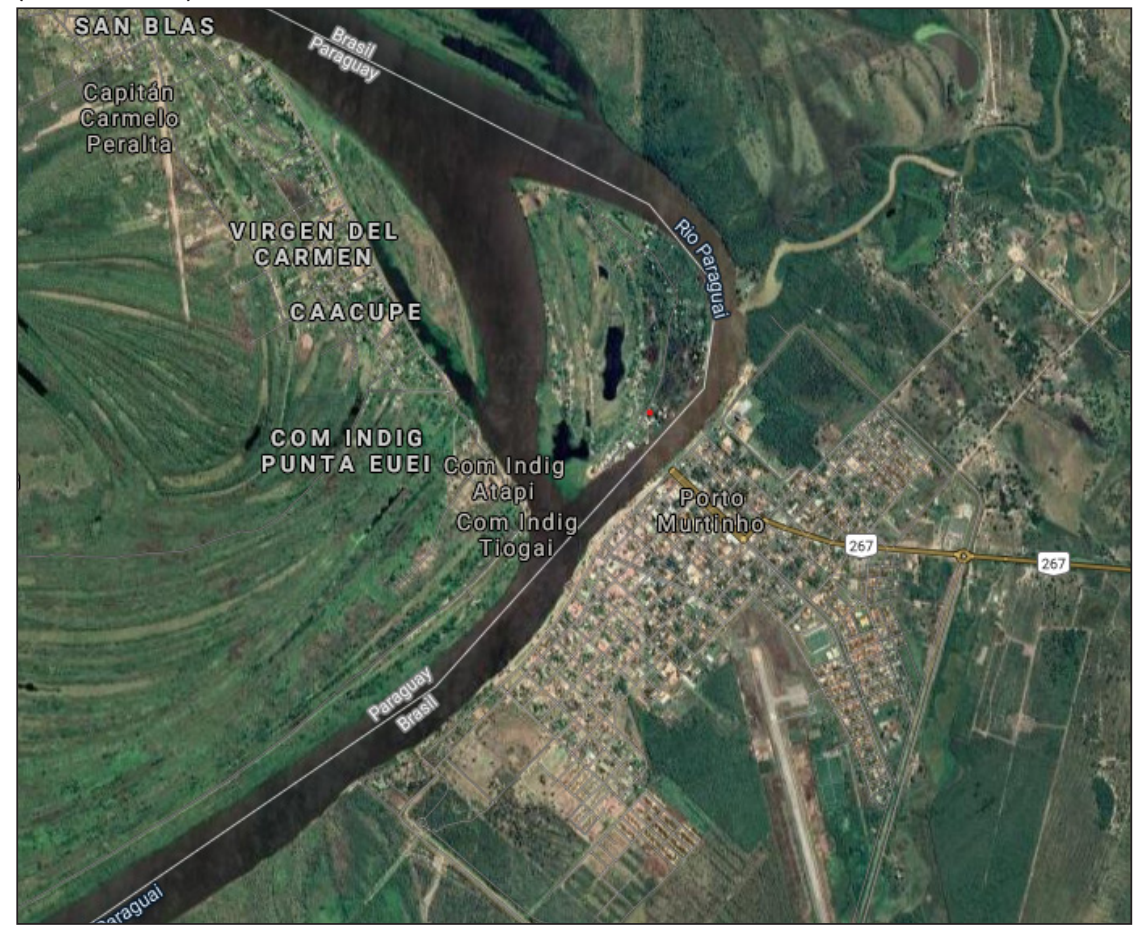

Fuente: Google (2021).

Este simbolismo se observa en fiestas, comidas y costumbres practicadas en común por la población de las dos ciudades. Una de las costumbres más tradicionales y practicadas en común se vive a orillas del río Paraguay, en los meses de octubre y noviembre, con el festival de pesca denominado "Festival Internacional de Pesca", cuando cada embarcación porta la bandera de su país como símbolo de distinción entre ellos. Sin embargo, el momento se vive en conjunto sin dar espacio a la exclusión sentimental.

En diciembre se celebra la mayor fiesta de devoción y unión entre los pueblos, la celebración de Nuestra Señora de Caacupé, patrona virgen santa de Paraguay, pero que es celebrada por el pueblo brasileño debido al sentido de pertenencia desarrollado por los vecinos de Porto Murtinho-BR.

En términos de alimentación, las ciudades también han agregado una herencia cultural de la otra, puesto que el tereré y el coquito también están presentes en la culinaria de los dos países, así como la danza y las músicas paraguayas son comúnmente practicadas por el pueblo brasileño, bien como el Carnaval es celebrado por los paraguayos, y eso sucede de forma armoniosa y relajada en la frontera.

\section{LA CULTURA PARAGUAYA}

Paraguay es un país donde la tradición indígena se mezcló con los rasgos de las civilizaciones europeas. Se trata, entonces, de una nación culturalmente única, que combina los elementos de la modernidad con las fuertes raíces de los pueblos ancestrales. Tanto es así que cuenta con dos 
idiomas oficiales: el guaraní, de origen indígena, y el español, legado de sus colonizadores, según lo dispuesto en el Art. 140 de la Constitución de la República del Paraguay (PARAGUAY, 1992).

De esta manera, "[...] el uso de la lengua guaraní, más allá de los grupos pertenecientes al pueblo guaraní, lo cual influyó en la identidad nacional y en el desarrollo de las expresiones artísticas como la poesía, el teatro y la música" (PARAGUAY, 2019a, p. 14).

La Resolución n. 869/2019 de la Secretaría Nacional de Cultura de Paraguay, que aprobó el Plan Nacional de Cultura 2018-2023, establece que:

La cultura se construye en torno a los pilares de la identidad colectiva, la cohesión social y el sentido de proyecto común. Paraguay, desde sus orígenes ancestrales, es multicultural, multilingüe, y también tiene dos lenguas oficiales, de las que el guaraní es de origen indígena. Esta riqueza de los acervos patrimoniales nacionales culturales se comprueba en las diversas y múltiples manifestaciones y actividades culturales y artísticas. (PARAGUAY, 2019b, p. 2).

En este contexto, los idiomas hablados en la sociedad paraguaya revelan las características de su cultura y de su identidad. Atribuirle al guaraní el estatus de lengua oficial es reconocer y valorar a los ancestros indígenas del país, de la misma forma que indica su importancia e influencia en otros aspectos culturales de este pueblo.

En este tercer milenio, lograron llegar a los "más fuertes", los "más resistentes", los que mejor se adaptaron a los desafíos naturales y culturales de toda la humanidad. Uno de estos héroes que cruzó con éxito la línea que separa la vida de la muerte, es el idioma guaraní. Por ello, podemos afirmar que el guaraní es una lengua maravillosa, valiente y viva; lo que no que quiere decir que es la única. (OLIVEIRA, 2011, p. 21).

Si bien el guaraní es la lengua indígena más hablada, no es la única, ya que "[...] son muchas las lenguas que luchan por seguir existiendo, y muchos los hablantes que piensan, sienten y utilizan estos idiomas para descifrar el mundo" (GAONA, I., 2019, p. 11). Tal aspecto también se reconoce por la Constitución Paraguaya (PARAGUAY, 1992), que en su Art. 140 dispone que "las lenguas indígenas, así como las de otras minorías, forman parte del patrimonio cultural de la Nación".

Es cierto que "lejos de ser considerada un conjunto homogéneo, resultado de un trayecto lineal, la cultura desarrollada en Paraguay debe ser vista como un complejo de procesos distintos" (ESCOBAR, 2019, p. 19), forma que el rico escenario paraguayo cuenta con diversas vertientes culturales, algunas que reflejan la modernidad, de la misma manera que otras, siguen siendo fieles a las tradiciones de los pueblos ancestrales. En este sentido, y como no podía ser diferente, este estudio no pretende agotar un tema tan sensible y valioso y, respetuosamente, intenta presentar un panorama de esta realidad cultural multifacética.

Como bien salienta el Plan Nacional de Cultura 2018-2023, "En todo el territorio nacional, Paraguay cuenta con un acervo patrimonial cultural diverso, intercultural e importante: culturales y artísticos, históricos, contemporáneos, urbanos, rurales, religiosos, seglares, indígenas, entre otros" (PARAGUAY, 2019b, p. 4).

La gastronomía paraguaya origina del conocimiento gastronómico español con el uso de los productos locales, sin menospreciar "La valiosa contribución y conocimiento de los pueblos guaraníes, en un saber-hacer que fue heredado de generación en generación, y que se inscribe en un hito histórico cultural local, regional y universal" (GODOY, 2020, p. 121).

Tal y como señala la Embajada de la República de Paraguay en Portugal (PARAGUAY, 2017), los principales ingredientes de su culinaria son el maíz, la mandioca y el cacahuete, y los productos más conocidos son "el chipá, la sopa paraguaya el condói, el mbejú, del chipá, y el kaburé". 
Quizás el plato más característico del país sea la sopa paraguaya, que en realidad no es una sopa, sino una clase de torta a base de harina de maíz.

La sopa paraguaya es uno de alimentos típicos de la culinaria paraguaya. Es una especie de bizcocho salado elaborado con harina de maíz, queso, cebolla, huevos, aceite y sal. La harina de maíz utilizada se conoce en la región como harina de maíz o avati ku'í (en idioma guaraní), que se obtiene del avatí morotĩ (maíz blanco), una variedad de maíz muy utilizada en Paraguay, y se encuentra en prácticamente todas los establecimientos agrícolas del país. (KUKIEL; SILVEIRA, 2020, p. 206, traducción libre de los autores).

Otro producto típico es el chipá, una especie de pan salado, hecho "principalmente con almidón de mandioca, harina de maíz, grasa de cerdo, huevo, queso, leche, entre otros ingredientes que se combinan de manera variada teniendo como registro, hasta el año 2000, unas 70 recetas variantes" (GODOY, 2020, p. 122).

No se puede olvidar un manjar paraguayo más, a saber, la yerba mate, el «oro verde», es el legado de los guaraníes, y cumple, más allá de sus propiedades nutricionales, un rol social» (PARAGUAY, 2018). Esta yerba es la base de la bebida tradicional conocida como tereré, además de su uso en otras técnicas culinarias.

La Secretaría Nacional de Cultura de Paraguay (PARAGUAY, 2018) ha establecido algunos circuitos gastronómicos, a saber: el Ka'a Rape o Ruta de la Yerba Mate, dirigida a los interesados y amantes de la yerba mate, que pasa por las ciudades/distritos de Encarnación, Capitán Miranda, Nueva Alborada, Trinidad, Jesús, Hohenau, Obligado, Bella Vista y Capitán Meza; el Chipá Rapé, que se da a lo largo de la Semana Santa y pasa por las tiendas de chipá de las ciudades de Eusebio Ayala y Coronel Bogado; y la Ruta de la Caña Paraguaya, para los amantes de la tradicional bebida local, que se realiza en la ciudad de Piribebuy.

La artesanía paraguaya, de acuerdo con la Resolución n. 270/20 del Instituto Paraguayo de Artesanía (PARAGUAY, 2020), se clasifica en tres categorías: i) artesanía popular tradicional, que consiste en la producción de objetos a partir de materias primas de origen local, cuya técnica es transmitida de generación en generación, que están dotados de valor simbólico y cultural; ii) arte indígena, que se refiere a la producción que transmite valores culturales y de identidad de los pueblos indígenas; y, iii) la artesanía contemporánea, que se refiere a la re significación de la artesanía popular e indígena mediante el uso de técnicas modernas, con la prevalencia del valor y la identidad cultural local.

Para Bobadilla y Bate (2018), el trabajo artesano involucra procesos técnicos, productivos, económicos y culturales, que utilizan materias primas locales, generalmente provenientes de la naturaleza, que se transmiten de generación en generación. Citan como ejemplos en Paraguay, tejedoras del ñanduti (puntilla), las bordadoras de Ao Poi y las ceramistas de Tobati.

A lo largo del proceso artesanal, se entremezclan elementos técnicos y productivos que permiten al artesano utilizar sus propias manos o valerse de herramientas para la elaboración de los productos, a partir del ingenio o la creatividad, utilizando técnicas que generalmente fueron heredadas dentro del entorno familiar, que se transmiten de generación en generación y son visibles localmente. (BOBADILLA; BATE, 2018, p. 20-1).

El instituto Paraguayo de Artesanía (PARAGUAY, 2019c) presenta el arte indígena que se vale de fibras naturales, madera, semillas, plumas y otros materiales de la naturaleza. Y, junto al lado ñanduti, de Ao poi y de la cerámica, también apunta a la confección de joyas, arte en cuero, hamacas y el sombrero Pirí. 
Con relación a la música, señala Saúl Gaona (2019) que los géneros típicamente paraguayos son la polka, el rasguito doble, el valsado y la guarania, todos correspondientes a la «música popular siendo los tres primeros de origen folklórico y el último con paternidad reconocida y certificado de nacimiento: José Asunción Flores, 1925 (GAONA, S., 2019, p. 78).

También forman parte del acervo cultural de Paraguay: el Archivo Nacional de Asunción, fundado en 1541, que alberga documentos que tienen fecha del siglo XVI; la Biblioteca Nacional de Paraguay, fundada en 1887; y los museos Casa de la Independencia, Histórico Nacional General Bernardino Caballero, Nacional de Bellas Artes, Casa Oratorio Cabañas, Dr. Gaspar Rodríguez de Francia y Cabildo de Pilar.

A su vez, el Municipio de Carmelo Peralta, ubicado en el Departamento de Alto Paraguay, tiene una población aproximada de 4.000 habitantes, conformada por latinos, indígenas etnia ayoreo y campesinos brasileños (PARAGUAY, 2021a). Constan en el Sistema Nacional de Información Cultural de Paraguay (PARAGUAY, 2021b), como festividades típicas de la ciudad, la fiesta patronal en honor a San Pedro, que se celebra el 9 de junio, la fiesta patronal en honor a la Virgen del Carmen, el 16 de julio, y la fiesta en homenaje a Santa Margarita, el 16 de octubre.

\section{LA CULTURA BRASILEÑA Y SUS RELACIONES FRONTERIZAS}

Los estudios sobre territorios e identidades culturales admiten hoy la integración multicultural, explicada anteriormente a través de la frontera simbólica y, más directamente, en las consideraciones de Vieira, Vieira y Knopp (2010), quienes afirman que "territorios plurales" y "pluralidad de territorios" conectados, resultan de la articulación de multiterritorialidades en un mismo espacio, haciendo múltiples lugares, híbridos o "el sentido global del lugar". Las identidades y manifestaciones culturales son intercambiables, al igual que el idioma, la economía y los insumos.

La cultura es la máxima expresión de la diversidad entre pueblos y razas, regiones y territorios. En cada rincón del territorio hay una manifestación de la cultura como producto del patrimonio histórico. Cultura local que interactúa con la cultura global a través de los medios globales que tan rápidamente ejercen influencia y crean poder. (VIEIRA; VIEIRA; KNOPP, 2010, p. 17, traducción libre de los autores).

Porto Murtinho es el municipio de Mato Grosso do Sul que forma frontera con Paraguay, delimitada por el río Paraguay, tras la Guerra de la Triple Alianza (1870). A través de la demarcación de fronteras, Tomás Laranjeira solicitó permiso para explorar los cultivos de yerba de la región, consolidando allí la prometedora economía de la yerba mate en la ex provincia de Mato Grosso.

Con la apertura de licitaciones públicas para el arrendamiento de los cultivos de yerba en 1892, surge una sociedad entre la familia Murtinho (Banco Rio y Mato Grosso) y Tomás Laranjeira, consolidando la empresa Cia. Mate Laranjeira y, en el mismo año, la construcción del puerto naval para la exportación de yerba mate a Paraguay, Argentina y a algunos países de Europa, fortaleciendo así la economía y los intercambios culturales entre los inmigrantes atraídos por el desarrollo de la región. A partir de entonces, el pueblo fue elevado a la categoría de villa y el 13 de junio de 1912, denominado municipio de Porto Murtinho (LIMA, 2000).

Tras el descubrimiento del tanino presente en la madera "Quebracho" en 1934, portugueses y alemanes instalaron fábricas para explotar esta materia prima para la fabricación de productos químicos y cueros.

INTERAÇÕES, Campo Grande, MS, v. 22, n. 4, p. 1175-1188, out./dez. 2021. 
Con el éxito de las dos empresas se produjeron grandes migraciones de brasileños del noreste, oeste de São Paulo y norte de Mato Grosso, provocando un aumento de población, que llamó la atención sobre el comercio, explotado por familias de Oriente Medio, la inmigración estaba sucediendo a nivel nacional. El municipio movía grandes ingresos y la economía estaba latente. Todo esto hizo que Porto Murtinho volviera a tener un lugar destacado en el escenario de desarrollo del Estado. (LIMA, 2000, p. 141, traducción libre de los autores).

La relación entre Brasil y Paraguay a través de Porto Murtinho se da no solo en las relaciones económicas e intercambios culturales, sino en la gastronomía y la música: chipa, sopa paraguaya, tereré y polka, sino también en la religiosidad, cuya mayor expresión es la devoción a Nuestra Señora de Caacupé, patrona de Paraguay y de Porto Murtinho.

[...] la formación poblacional del municipio de Porto Murtinho tiene en sus raíces el aporte cultural de las naciones: Kadiwéu, Terena y también los pueblos indígenas paraguayos Ayoréo, además de la contribución de los paraguayos que migraron a la ciudad. Así, todos estos pueblos indígenas y paraguayos están muy presentes en las manifestaciones culturales del pueblo murtinhense [...]. (HEYN, 2003, p. 56, traducción libre de los autores).

Porto Murtinho tiene, entonces, una relación cultural con Paraguay muy característica, representada por la Fiesta del Toro Candil, una atracción folklórica en Paraguay, adaptada por los vecinos de Porto Murtinho. Castilho y Paiva (2013) relatan la leyenda paraguaya que cuenta el cuento del Toro Candil, que cruzó el río Paraguay y se enamoró de la vaquilla Estrella, dando a la luz al toro Bandido, valeroso y fuerte, bendecido por Nuestra Señora de Caacupé.

Así nació la fiesta en Porto Murtinho, que es una mezcla de religiosidad y folklore que involucra la Leyenda del Toro Candil. Durante la presentación, los dos toros se desafían a ver quién es el hijo legítimo del Toro Candil. Los toros, Bandido y Encantado, están representados por el color verde y amarillo, respectivamente. Los colores que también denotan los tonos principales de la Bandera Nacional demuestran los lazos culturales entre Brasil y Paraguay, los cuales están divididos solo por un río. (CASTILHO; PAIVA, 2013, p. 60, traducción libre de los autores).

Desde 2005, la fiesta se lleva a cabo con la participación de adolescentes en el montaje y la puesta en escena. En este contexto, Castilho y Paiva (2013, p. 63, traducción libre de los autores) destacan que "[...] I patrimonio cultural urbano es una construcción simbólica del imaginario popular en la frontera" $y$, en este caso, existe una interculturalidad entre los territorios. Las actividades turísticas del municipio incluyen fiestas, rutas navales y concursos de pesca en el Pantanal Nabileque (PORTO MURTINHO, 2016).

Heyn (2003) también informa que la población de Carmelo Peralta y otras ciudades de Paraguay impactadas por las crecidas del río Paraguay, alternaron residencia en busca de refugio en Porto Murtinho tras la construcción del dique en la década de 1980, y que esta comunidad se convirtió en parte de la población local formando el barrio Caacupé.

Porto Murtinho tiene ahora casi 17.300 habitantes (IBGE, 2020) y limita con la ciudad de Carmelo Peralta (PY) a través de pequeños transbordadores. La construcción del puente entre estas ciudades permitirá un mayor flujo de carga y personas, profundizando las relaciones culturales entre ellas, además de transformar la multiculturalidad territorial muy dinámica de ese lugar.

\section{CONSIDERACIONES FINALES}

Las discusiones sobre territorialidad, identidad y fronteras simbólicas traen reflexiones sobre los intercambios culturales a través de una caracterización de la multiculturalidad. Entonces, 
se entiende que la presencia de múltiples territorios en un espacio culturalmente plural, con identidades únicas y economías específicas, traen profundos consecuencias a las comunidades fronterizas.

Paraguay, con su tradición indígena y mezclas de rasgos de las civilizaciones europeas, se ha convertido en una nación culturalmente singular, especialmente en cuanto a los idiomas que se hablan allí, herencia de sus colonizadores. Porto Murtinho, por su parte, está marcado por los diferentes ciclos económicos de desarrollo que ocurridos en su territorio, el ciclo de la yerba mate, del tanino y de la cecina. Así, a lo largo de esta investigación se identificó que, a pesar de las peculiaridades de cada territorio, marcados por procesos de colonización específicos, cuando se encuentran al otro lado de la frontera, acaban por constituir una nueva cultura e identidad, muy propia de ese espacio construido. Además, las ciudades tienen rasgos comunes como impactos naturales, acciones económicas y creencias religiosas.

El Corredor Bioceánico, además de profundizar estas interacciones y relaciones, muy probablemente ampliará estos límites y mestizaje. Porto Murtinho, en Brasil, y Carmelo Peralta, en Paraguay, ya son ciudades gemelas y activas entre sí, y a pesar de sus especificidades, tienen una cultura común, una economía rotatoria y una historia compartida. El puente materializa la simbología de la frontera que ya existe en ese territorio, profundizando aún más estos intercambios culturales al eliminar la barrera fronteriza del río.

Es posible, entonces, suponer que, a partir de la consolidación del puente, esta unificación será amplificada y se convertirá cada vez más multicultural en el territorio, ya que la frontera como elemento simbólico se convierte en productora del sentimiento de pertenencia, y a través de ella se desarrolla en uno de los pilares de la identidad, es decir, es en la frontera donde el territorio se divide y se unifica imperceptiblemente.

\section{REFERENCIAS}

ASATO, Thiago Andrade; GONÇALVES, Débora Fittipaldi; WILKE, Erick Pusck. Perspectivas del Corredor Bioceánico para el desarrollo local en el estado de MS: el caso de Porto Murtinho. Interações, Campo Grande, v. 20, número especial, p. 141-57, 2019.

BENEDICT, Anderson. Comunidades imaginadas: reflexões sobre a origem e a difusão do nacionalismo. 4. reimp. Tradução de Denise Bottman. São Paulo: Companhia das Letras, 2008.

BOBADILLA, Fabiola Sánchez; BATE, Santiago Galeano. La artesanía y su relación con el turismo. Revista Científica OMNES de la Universidad de Columbia del Paraguay, Asunción, v. 1, n. 2, p. 18-27, 2018.

CAMPOS, Luana; FARIA, Alcides. Rota Bioceânica: o que é e seus impactos diretos e indiretos. Ecoa, [s.l.], 2020. Disponible en: https://ecoa.org.br/rota-bioceanica-o-que-ee-seus-impactos-diretos-e-indiretos/. Acceso el: 25 abr. 2021.

CASTILHO, Maria Augusta; PAIVA, Nivea Maria Mendes. Patrimônio cultural na festa do touro candil em Porto Murtinho, MS. Multitemas, Campo Grande, n. 43, p. 53-65, 2013. Disponible en: https://www. multitemas.ucdb.br/multitemas/article/view/264/309. Acceso el: 20 jun. 2021.

ESCOBAR, Ticio. La cultura en el Paraguay: los tiempos de lo diverso. In: SIMPOSIO DE MÚSICA EN PARAGUAY: SITUACIÓN ACTUAL Y PERSPECTIVAS DE FUTURO, 1., jul. 2019, Asunción. Anais [...]. Asunción: Cultura Paraguay, 2019. p. 19-32. Disponible en: http://www.cultura.gov.py/documentos/la-musica-enel-paraguay-situacion-actual-y-perspectivas-de-futuro/. Acceso el: 10 mayo 2021. 
GAONA, Ivonne (Coord.). El pensamiento que fluye en las lenguas indígenas vivas en Paraguay. Asunción: Ministerio de Educación y Ciencias, 2019. Disponible en: http://www.cultura.gov.py/documentos/elpensamiento-que-fluye-en-las-lenguas-indigenas-vivas-en-paraguay/. Acceso el: 11 mayo 2021.

GAONA, Saúl. La música popular en el Paraguay, desde la colonia hasta el presente. In: SIMPOSIO DE MÚSICA EN PARAGUAY: SITUACIÓN ACTUAL Y PERSPECTIVAS DE FUTURO, 1., jul. 2019, Asunción. Anais [...]. Asunción: Cultura Paraguay, 2019. p. 73-89. Disponible en: http://www.cultura.gov.py/documentos/ la-musica-en-el-paraguay-situacion-actual-y-perspectivas-de-futuro/. Acceso el: 10 mayo 2021.

GODOY, Zulma Inés Masi. El chipá y su estampa femenina: memoria, tradición, trabajo y patrimonio. In: MARCHI, Darlan de Mamman; KNACK, Eduardo Roberto Jordão; POLONI, Rita Juliana Soares (Org.). Memória \& Patrimônio: identidade, emoção e ditaduras. Pelotas: Editora UFPel, 2020. p. 120-37. v. 2.

GOOGLE. Frontera Brasil-Paraguay Porto Murtinho-Carmelo. Google Maps, [s.I.], 2021. Acceso el: 28 jun. 2021.

HEYN, Carlos Alberto. Desenvolvimento local endógeno: análise de experiência em Porto Murtinho -MS. 2003. Dissertação (mestrado em Desenvolvimento Local) - Universidade Católica Dom Bosco, Campo Gande, MS, 2003. Disponible en: https://site.ucdb.br/public/md-dissertacoes/7949-desenvolvimentolocal-endogeno-analise-de-experincia-em-porto-murtinho-ms.pdf. Acceso el: 20 jun. 2021.

IBGE. Panorama: Porto Murtinho. IBGE, [s.I.], 2020. Disponible en: https://cidades.ibge.gov.br/brasil/ms/ porto-murtinho/panorama. Acceso el: 28 jun. 2021.

KUKIEL, Éder Damião Goes; SILVEIRA, Claudia Vera. A culinária de fronteira como elemento de união entre povos: o caso da sopa paraguaia na fronteira entre Brasil e Paraguai e Brasil e Bolívia. Boletim Gaúcho de Geografia, Porto Alegre, v. 47, n. 1, p. 201-224, 2020.

LIMA, Maria Margareth Escobar Ribas. Ciclos econômicos e produção arquitetônica em Porto Murtinho. Campo Grande, MS: Life Editora, 2013.

LIMA, Maria Margareth Escobar Ribas. O resgate de Porto Murtinho. Ensaios e Ciência: Ciencias Biológicas, Agrárias e de la Saúde, Campo Grande, v. 4, n. 3, p. 135-43, 2000. Disponible en: https://www.redalyc. org/articulo.oa?id=26040309. Acceso el: 20 jun. 2021.

MATO GROSSO DO SUL. VIII Reunião do Corredor Rodoviário Bioceânico: evento. Corredor Bioceânico, [s.l.], 2019. Disponible en: http://www.corredorbioceanico.ms.gov.br/oevento/. Acceso el: 25 de abril. 2021.

OLIVEIRA, David Galeano. El idioma y cultura guaraní en Paraguay. [S.I.]: Lulu, 2011.

PARAGUAY. Ministerio de Tecnologías de la Información y las Comunicación. Municipalidad Carmelo Peralta. Municipios República del Paraguay, Capitán Carmelo Peralta, 2021a. Disponible en: https://www. municipios.gov.py/carmeloperalta/. Acceso el: 10 mayo 2021.

PARAGUAY. Sistema Nacional de Información Cultural del Paraguay, 2021b. Disponible en: http://sicpy. gov.py/. Acceso el: 10 mayo 2021.

PARAGUAY. Resolución n. 270/20. Registros de artesano. Asunción, 2020. Disponible en: https://www. artesania.gov.py/index.php/tramites/registros-y-tramites. Acceso el: 11 mayo 2021.

PARAGUAY. Secretaría Nacional de Cultura. La música en el Paraguay: situación actual y perspectivas de futuro. In: SIMPOSIO DE LA MÚSICA EN EL PARAGUAY, 1., jul. 2019, Asunción. Anais [...]. Asunción: Cultura Paraguay, 2019a. Disponible en: http://www.cultura.gov.py/documentos/la-musica-en-el-paraguaysituacion-actual-y-perspectivas-de-futuro/. Acceso el: 10 mayo 2021. 
PARAGUAY. Resolución n. 869/2019. Por el cual se aprueba el Plan Nacional de Cultura 2018-2023 de la Secretaría Nacional de Cultura en idioma guaraní. Asunción, 2019b. Disponible en: http://sicpy.gov.py/ generales/?7149\#Plan\%20Nacional\%20de\%20Cultura\%20en\%20Guaran\%C3\%AD. Acceso el: 10 mayo 2021.

PARAGUAY. Ministerio de Tecnologías de la Información y Comunicación. Instituto Paraguayo de Artesanía. Artesanía, Asunción, 2019c. Disponible en: https://www.artesania.gov.py/index.php/tramites/artesania. Acceso el: 10 mayo 2021.

PARAGUAY. Secretaría Nacional de Turismo. Ka'a Rape. Asunción, 2018. Disponible en: https://visitparaguay. travel/v1/circuito/4-kaa-rape. Acceso el: 10 mayo 2021.

PARAGUAY. Ministerio de Relaciones Exteriores. Cultura. Embaixada do Paraguai, Lisboa, 2017. Disponible en: https://embaixadadoparaguai.pt/pt-pt/cultura/\#toggle-id-1. Acceso el: 10 mayo 2021.

PARAGUAY. Constituición Nacional de la República del Paraguay. Asunción, 1992. Disponible en: https:// www.bacn.gov.py/constitucion-nacional-de-la-republica-del-paraguay. Acceso el: 10 mayo 2021.

PESAVENTO, Sandra Jatahy. Além das fronteiras. In: MARTINS, Maria Helena (Org.). Fronteiras culturais - Brasil, Uruguai, Argentina. Cotia, SP: Ateliê editorial, 2002. p. 35-39.

PORTO MURTINHO. Guia de turismo. Prefeitura de Porto Murtinho, 2016. Disponible en: http://www. portomurtinho.ms.gov.br/guia-turismo. Acceso el: 20 junio 2021.

SOUZA, Mariana Jantsch. Fronteiras simbólicas: espaço de hibridismo cultural, uma leitura de dois irmãos, de Milton Hatoum. Revista Letrônica, Porto Alegre, v. 7, n. 1, p. 475-89, 2014.

VIEIRA, Marcelo Milano Falcão; VIEIRA, Eurípedes Falcão; KNOPP, Glauco da Costa. Espaço global: território, cultura e identidade. Revista Administração em Diálogo, São Paulo, v. 12, n. 2, p. 1-19, 2010.

\section{Sobre las autoras:}

Hanae Caroline Quintana Shiota: Estudiante de Máster en el Programa de Posgrado en Desarrollo Local de la Universidad Católica Dom Bosco (UCDB). Posgrado en Derecho Procesal Civil por la Facultad de Derecho Prof. Damásio de Jesus. Grado en Derecho por la UCDB. Miembro del Grupo de Investigación sobre Patrimonio Cultural, Derechos y Diversidad. Investigadora y coasesora de iniciación científica del Proyecto de Investigación Ruta Bioceánica y UniRila (Universidades de la Ruta de la Integración Latinoamericana): Conociendo el Potencial Existente como Nuevos Espacios de Desarrollo y Práctica. E-mail: hanae_shiota@hotmail.com, Orcid: https://orcid.org/0000-0002-6841-7334

Mariana de Barros Casagranda Akamine: Estudiante de Máster en Desarrollo Local en la Universidad Católica Dom Bosco (UCDB). Posgrado en Diseño de Interiores por la UCDB; y en Rehabilitación Ambiental Sostenible Arquitectónica y Urbana por la Universidad de Brasilia (UnB). Arquitecta y urbanista por la Universidad Anhanguera-UNIDERP. Actualmente, es profesora en el Centro Universitario da Grande Dourados (UNIGRAN) - Capital, en el curso de Arquitectura y Urbanismo.

E-mail: mariana_casagranda@yahoo.com.br, Orcid: http://orcid.org/0000-0001-9605-1061

Alessandra Chaia: Estudiante de Máster en Desarrollo Local en la Universidad Católica Dom Bosco (UCDB). Grado en Arquitectura y Urbanismo por el Centro de Educación Superior Prof. Plínio 
Mendes dos Santos (CESUP). Profesora en el Centro Universitario da Grande Dourados (UNIGRAN) - Capital. E-mail: alessandrachaia@gmail.com, Orcid: https://orcid.org/0000-0002-7087-5238

Éricka Santos Silva: Estudiante de Doctorado en Desarrollo Local en el Contexto de Territorialidades en la Universidad Católica Dom Bosco (UCDB). Máster en Desarrollo Local en el Contexto de Territorialidades por la UCDB. Grado en Artes Visuales por la Universidad Federal de Mato Grosso do Sul (UFMS); y en Arquitectura y Urbanismo por la Universidad Anhanguera-UNIDERP. Miembro del Grupo de Investigación en Patrimonio Cultural, Derecho y Diversidad (CNPq). E-mail: erikasantos.arqdeco@gmail.com, Orcid: https://orcid.org/0000-0003-3715-8015

Arlinda Cantero Dorsa: Realizando prácticas postdoctorales en Desarrollo Local en el Centro Universitario Augusto Motta (UNISUAM). Doctorado en Lengua Portuguesa por la Pontificia Universidad Católica de São Paulo (PUC-SP). Máster en Comunicación y Letras por la Universidad Presbiteriana Mackenzie (UPM). Grado en Letras/Francés por la Facultad Dom Aquino de Filosofía, Ciencias y Letras; y en Pedagogía por la Facultad de Educación, Ciencias y Letras de Urubupungá (FECLU). Profesora titular de la Universidad Católica Dom Bosco (UCDB), en el curso de Derecho. Profesora y vicecoordinadora del Máster y Doctorado en Desarrollo Local de la UCDB. Miembro del Instituto Histórico e Geográfico de Mato Grosso do Sul (IHG-MS). Líder del Grupo de Investigación en Patrimonio Cultural, Derecho y Diversidades y subdirectora del Grupo de Investigación y Estudios en Tecnología Educativa y Educación a Distancia (GETED) de la UCDB. Coordina el proyecto Ruta Bioceánica y UniRila (Red Universitaria de la Ruta de Integración Latinoamericana): Conociendo el Potencial Existente como Nuevos Espacios de Desarrollo y Prácticas Innovadoras - Continuidad; y el Proyecto Gobernanzas Migratorias en Brasil: Desafíos Contemporáneos en la Agenda 2030 - Organización de las Naciones Unidas (ONU). E-mail: acdorsa@ucdb.br, Orcid: http://orcid.org/0000-0002-1120-0273 